\title{
ACCURATE COLD-TEST MODEL OF HELICAL TWT SLOW-WAVE CIRCUITS
}

\author{
Carol L. Kory, Member, IEEE \\ Analex Corporation/NASA Lewis Research Center \\ 21000 Brookpark Rd., MS 54-5 \\ Cleveland, Ohio 44135 USA \\ phone: (216)-433-3512 email: Carol.L.Kory@lerc.nasa.gov \\ James A. Dayton, Jr., Senior Member, IEEE \\ NASA Lewis Research Center \\ 21000 Brookpark Rd., MS 54-5 \\ Cleveland, Ohio 44135 USA \\ phone: (216)-433-3515 email: jdayton@lerc.nasa.gov
}

Abstract Recently, a method has been established to accurately calculate cold-test data for helical slow-wave structures using the three-dimensional electromagnetic computer code, MAFIA [1]. Cold-test parameters have been calculated for several helical travelingwave tube (TWT) slow-wave circuits possessing various support rod configurations, and results are presented here showing excellent agreement with experiment. The helical models include tape thickness, dielectric support shapes and material properties consistent with the actual circuits. The cold-test data from this helical model can be used as input into large-signal helical TWT interaction codes making it possible, for the first time, to design a complete TWT via computer simulation.

\section{INTRODUCTION}

The analysis, including cold-test calculations, for helical slow-wave structures has been a long standing problem within the traveling-wave tube (TWT) industry. Although the helix slow- 
wave circuit remains the mainstay of the TWT industry because of its exceptionally wide bandwidth, a full helical circuit, without significant dimensional approximations, had not been successfully modeled until recently. Numerous attempts had been made to analyze the helical slow-wave circuit so that the performance could be accurately predicted without actually building it, but because of its complex geometry, many geometrical approximations became necessary rendering the previous models inaccurate. Ward et. al [2] developed a threedimensional model which included the actual helical dimensions without geometrical approximations. The model provided fairly good agreement between measured and simulated dispersion (within 5\%), but did not include a comparison of impedance or attenuation. In the course of this research it has been demonstrated that using the simulation code, MAFIA (Solution of MAxwell's equations by the Finite-Integration-Algorithm) [3, 4], the helical structure can be modeled with actual tape width and thickness, dielectric support rod geometry and materials. To demonstrate the accuracy of the MAFIA model, the cold-test parameters including dispersion, on-axis interaction impedance and altenuation have been calculated for several helical TWT slow-wave circuits with a variety o: support rod geometries including rectangular and $\mathrm{T}$-shaped rods, as well as various support rod materials including isotropic, anisotropic and partially metal coated dielectrics. Compared with experimentally measured results, the agreement is excellent.

\section{ANALYSIS AND SIMULATION}

The dispersion relation was calculated using a method similar to experimental methods in which the frequency-phase dispersion characteristics are calculated by measuring the resonant frequencies in a truncated circuit section. Here either a perf ct electric wall or a perfect magnetic 
wall is placed at each axial end of the circuit section [5]. The resonances correspond to standing waves that occur when the circuit length is an integral number of half wavelengths (phase shifts of $\pi$ ). Although the helix does not possess the reflection symmetry that allows total reflection of a single mode in a transverse conducting plane, useful data can be obtained by resonating the structure despite reactive end effects, as suggested by Nalos [6] and Watson and Whinnery [7]. To establish the number of turns which must be modeled for negligible end effects, several models were simulated with varying numbers of turns, keeping the mesh size proportionally constant. It was found that about eight turns are needed for converging results as shown in Figure 1 where phase velocity versus frequency is plotted for several circuit sections of varying number of turns for a C-band helical circuit. The MAFIA code possesses the capability to apply quasi-periodic boundary conditions to the circuit where a fixed phase advance per turn in the axial direction can be specified, and most of the computations presented in this paper were duplicated using this method. In every case the use of quasi-periodic boundary conditions increased the computing time by a factor of about two, while achieving comparable agreement with measured values. Details of these computations would only lengthen the paper without adding useful content; therefore, they are not included here. 


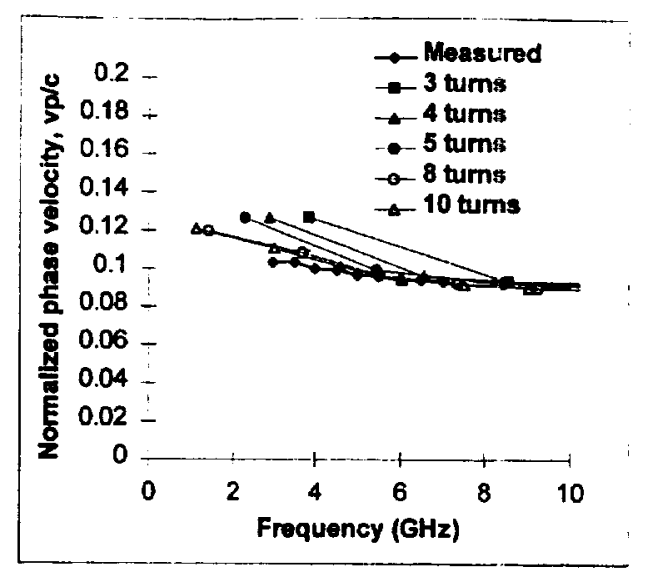

Figure 1 Phase velocity versus frequency for several circuit sections of varying number of turns for the Northrop C-band helical circuit

The on-axis interaction impedance is a measure of the strength of the coupling between the $\mathrm{RF}$ wave and the electron beam, defined for the $\mathrm{n}^{\mathrm{th}} \mathrm{RF}$ space harmonic as

$$
K_{n}=\frac{\left|E_{n}^{2}\right|}{2 \beta_{n}^{2} P_{R F}}
$$

[8], where $\left|E_{a}\right|$ is the axial electric field magnitude of the $n^{\text {th }}$ space harmonic. $\beta_{a}$ is the axial phase constant of the $\mathrm{n}^{\text {th }}$ harmonic defined by

$$
\beta_{n}=\frac{\phi+2 \pi n}{L},
$$

where $\phi$ is the phase shift per turn in radians of the fundamental harmonic, $n$ is the space harmonic order and $\mathrm{L}$ is the length of one turn. $\mathrm{P}_{\mathrm{RF}}$ is the time averaged $\mathrm{RF}$ power flow defined by

$$
P_{R F}=w v_{g},
$$

where $v_{g}$ is the group velocity and $w$ is the time averaged stored electromagnetic energy per unit length, 


$$
w=\frac{W_{T}}{N L},
$$

with $W_{\mathrm{T}}$ the total energy in the $\mathrm{N}$ turns. Amplification requires synchronism of the electron beam with the fundamental space harmonic; therefore, the fundamental $(n=0)$ harmonic term is used in (1) to determine the on-axis interaction impedance.

The circuit attenuation is calculated using the same truncation method as with the dispersion and impedance calculations. The attenuation, $\alpha$, is defined in $\mathrm{dB}$ per unit length as

$$
\alpha=8.686 \frac{P_{L}}{2 P_{R F}}
$$

[9], where $P_{L}$ is the total power loss per unit length,

$$
P_{L}=\frac{P_{L T}}{N L},
$$

and $P_{L T}$ is the total power loss for a traveling wave in the $N$ turns.

The versions of MAFIA (3.20 and 4.0) used in this work do not posses the capability to generate a helical structure automatically. Thus, the helix was generated in the cylindrical coordinate system by varying axial and azimuthal coordinates consistent with the formula of a circular helix.

\section{RESULTS}

The first circuit modeled is the helical slow-wave circuit from a Northrop Grumman C-Band TWT for the Microwave Power Module (MPM) [10]. The experimental circuit includes a rectangular, copper plated tungsten helical tape supported by rectangular $\mathrm{BeO}$ rods inside a conducting barrel as shown for the end view in Figure 2. Because the support rods do not conform to the cylindrical coordinate system, "quasi-rectangular" rods were modeled in radial 
increments by reducing the rod angles with increasing radius. The MAFIA three-dimensional plot of the circuit is shown in Figure 3.

The dispersion, attenuation and on-axis interaction impecance were calculated for this circuit using MAFIA and compared with measured results in Figure 4 and Figure 5. The agreement is excellent with absolute average differences across the bandwidth of $0.7 \%$ and six Ohms for the dispersion and impedance, respectively. The necessary MAFIA input for attenuation calculations includes an effective conductivity value and appropriate dielectric loss tangent. The loss tangent for $\mathrm{BeO}$ was taken to be .0001 . The effective conductivity value was taken from Gilmour et al. [11] where theoretical and experimental TWT helix loss was determined from about 2 to $16 \mathrm{GHz}$ for a copper plated helix. Gilmour et al. found excellent agreement between calculated and measured loss when helix surface roughness was assumed to cause the resistivity to increase by a factor of approximately two. Using a conductivity consistent with these results $\left(1.45 \times 10^{7} \mathrm{~S} / \mathrm{m}\right)$, the average absolute attenuation difference was about $.0005 \mathrm{~dB}$ per turn. The CPU time to simulate ten turns of this MAFIA C-band model is about 30 hours using an IBM RISC/6000 Model 590 Workstation. 


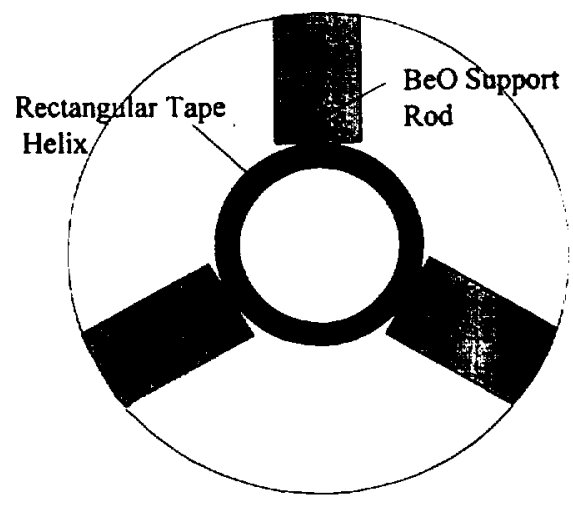

Figure 2 Northrop C-Band helical TWT slow-wave circuit

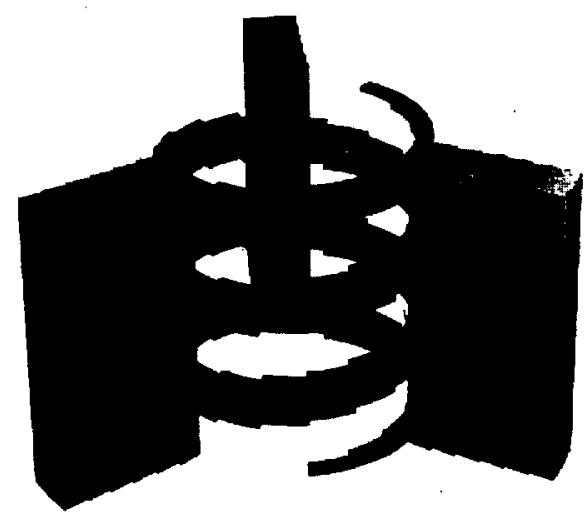

Figure 3 MAFIA three-dimensional plot of Northrop C-Band helical circuit 


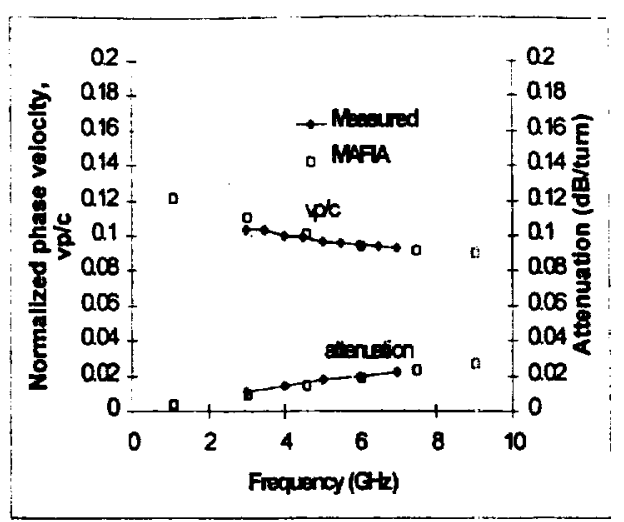

Figure 4 Measured and simulated dispersion and attenuation for Northrop C-Band helical circuit

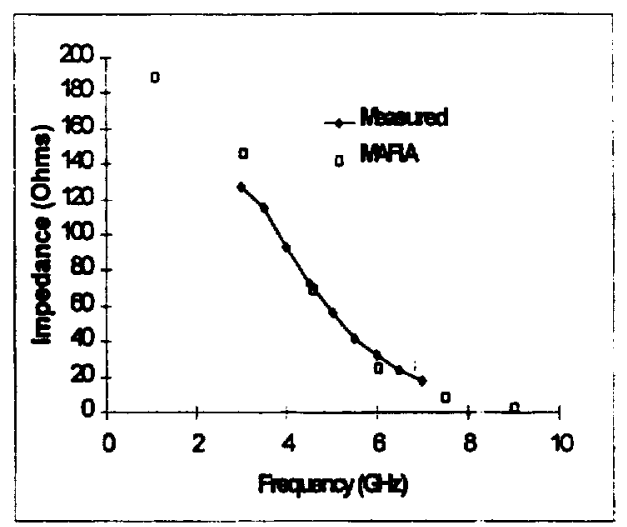

Figure 5 Measured and simulated on-axis interaction impedance for Northrop C-Band helical circuit

A Northrop Grumman broadband helical slow-wave circuit was also modeled using MAFIA, and the dispersion, on-axis interaction impedance and attenvation were calculated and compared with measured results [10]. The circuit, shown in Figure 6, embodies a tungsten/rhenium tape helix supported by rectangular $\mathrm{BeO}$ rods, with a copper film partially coating the support rods to broadband the circuit. Initially, the support rods were modeled using the quasi-rectangular configuration discussed previously, but with this configuration it is necessary to use ten radial 
increments for the dielectric support rods to prevent the protrusion of the dielectric material through the metal coating. This increased number of radial increments, compared to six for the C-Band circuit, complicates the boundary conditions of the problem and increases the time for the computation to converge. Because of this added complexity and computational time, the support rods were generated in wedges using graded effective dielectric constants [12]. It was shown in [12] that there was a significant savings in computational time with negligible change in dispersion and on-axis interaction impedance when using this modeling configuration. Figure 7 shows the cross-sectional view of the Northrop broadband circuit model with graded wedges. The wedges are modeled using four sections, and thus four effective dielectric constants.

The dispersion, attenuation and on-axis interaction impedance were calculated for this circuit using MAFIA and compared with measured results in Figure 8 and Figure 9. Compared to measured data the absolute average differences across the bandwidth are $1.5 \%, .003 \mathrm{~dB} /$ turn and ten Ohms, for the dispersion, attenuation and impedance, respectively.

The conductivity of the tape was initially approximated to be consistent with twice the resistivity of the theoretical value for tungsten, in line with [11] as described previously. The conductivity of the copper loading film was also kept consistent with twice the resistivity of the theoretical value for copper. When these values were used, the attenuation obtained using MAFIA was lower than experimental data. Using a value 2.46 times the theoretical resistivity value of tungsten for the helical tape gave the best agreement with measured attenuation as shown in Figure 8. 


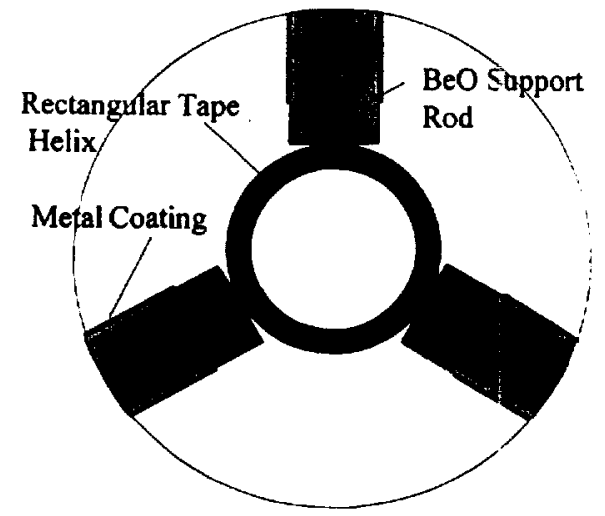

Figure 6 Northrop broadband helical TWT slow-wave circuit

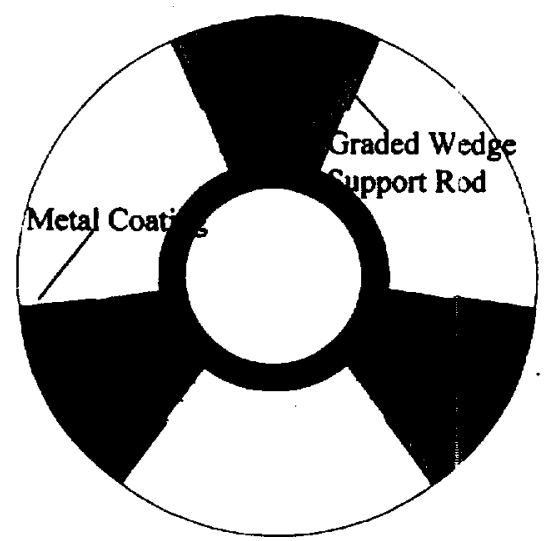

Figure 7 Northrop broadband helical slow-wave circuit model with graded wedge support rods 


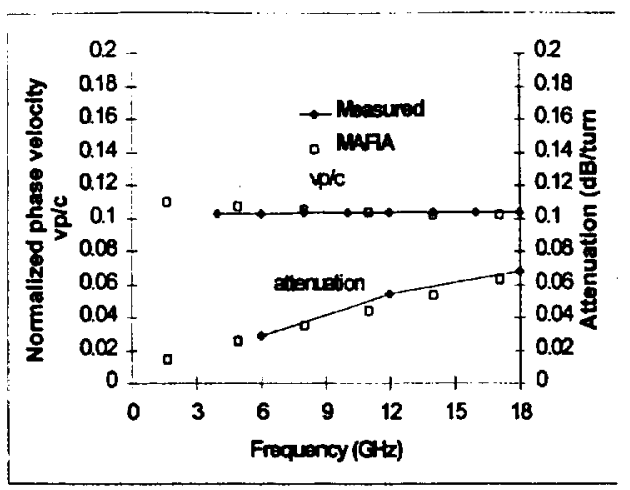

Figure 8 Measured and simulated dispersion and attenuation for Northrop broadband helical circuit

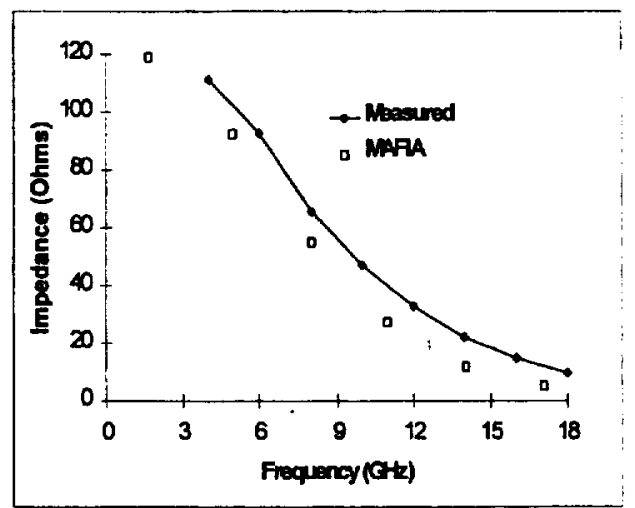

Figure 9 Measured and simulated on-axis interaction impedance for Northrop broadband helical circuit

Next, to demonstrate how MAFIA can be used to study circuits having more complex material characteristics, a circuit with anisotropic pyrolytic boron nitride (APBN) support rods was modeled. The circuit embodies a rectangular, copper plated tungsten/rhenium, helical tape supported by rectangular APBN rods oriented for low RF losses rather than thermal conductivity. The helical slow-wave circuit is from a $10 \mathrm{Watt}, 32 \mathrm{GHz}$ traveling-wave tube amplifier (TWTA) for incorporation into the Ka-Band Transmitter package for the Cassini mission [13]. It 
resembles the Northrop Grumman C-Band circuit of Figure 2. Similarly, the support rods were generated in the cylindrical coordinate system by using six radial increments by reducing the rod angle with increasing radius, or the quasi-rectangular configuration. Only the measured dispersion and attenuation were available from the manufacturer for comparison, which are compared to the values obtained using MAFIA in Figure 10.

For this circuit with the APBN support rods, the nominal dielectric constant is 5.1 parallel to the layers and 3.4 perpendicular. Since the rods are oriented for low RF losses rather than for thermal conductivity, this implies an effective dielectric constant of 5.1 in the azimuthal and axial directions, and 3.4 in the radial direction. Using these nominal values, the dispersion obtained using MAFIA was consistently lower than measured values by about one $\mathrm{GHz}$, or an absolute frequency difference of about $3 \%$. By varying the relative dielectric constant of the rods to 4.6 in the azimuthal and axial directions, and 2.3 in the radial direction, the results shown in Figure 10 were obtained. The average absolute difference across the bandwidth between measured dispersion and that obtained using MAFIA is only $0.6 \%$.

Some in-house measurements of APBN showed that the permittivity can vary within the same plate of material from the same manufacturer by as much as $+1-10 \%$ [14]. With this information, the variations made from the nominal permittivity values for the Cassini support rods are approximately justified. Unfortunately, it is impossible to establish the exact anisotropic permittivity of the dielectric rods used in these already assembled tubes.

The attenuation was calculated for the Cassini circuit using a conductivity value of $2.25 \times 10^{7}$ $\mathrm{S} / \mathrm{m}$ for the helical tape, nearly the theoretical value of pure copper, and compared with measured values in Figure 10. Much attention was given to polishing the helical tape to a bright, shining finish, therefore explaining this large conductivity value. 


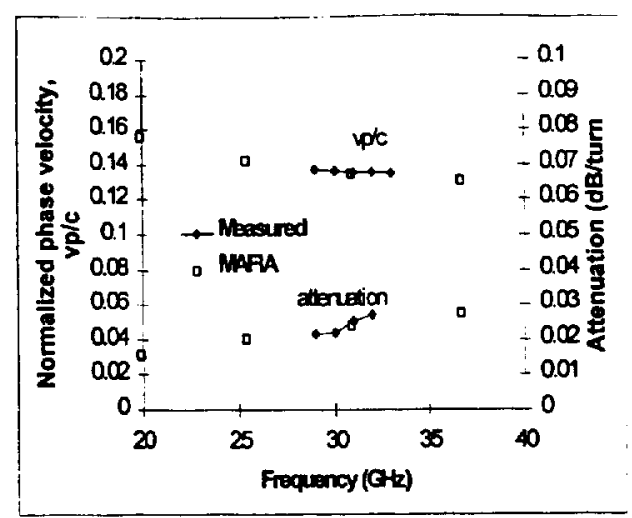

Figure 10 Measured and simulated dispersion and attenuation for Cassini helical circuit

The last circuit modeled was the helical slow-wave circuit from a Hughes TWT for the MPM [15]. The experimental circuit includes a rectangular, copper plated, helical tape supported by Tshaped $\mathrm{BeO}$ rods inside a conducting barrel as shown for the end view in Figure 11. Only the measured dispersion values were available from the manufacturer for comparison which are compared with the values obtained using MAFIA in Figure 12. The results using MAFIA agree very well with measured data with an absolute average difference across the bandwidth of $1.8 \%$.

As a result of the expressed preference of many industrial colleagues, dispersion relations are presented in this paper in the form of normalized phase velocity versus frequency, rather than the classic $\omega-\beta$ diagrams. Many of these dispersion relations can be found in the classic format in [12]. 


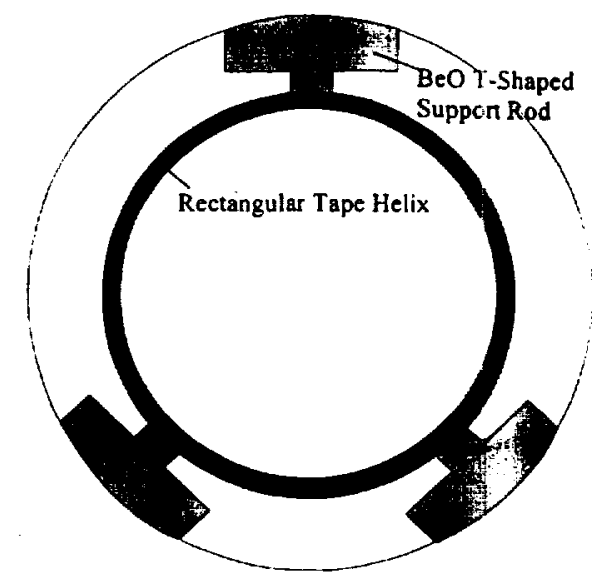

Figure 11 Hughes MPM helical slow-wave circuit

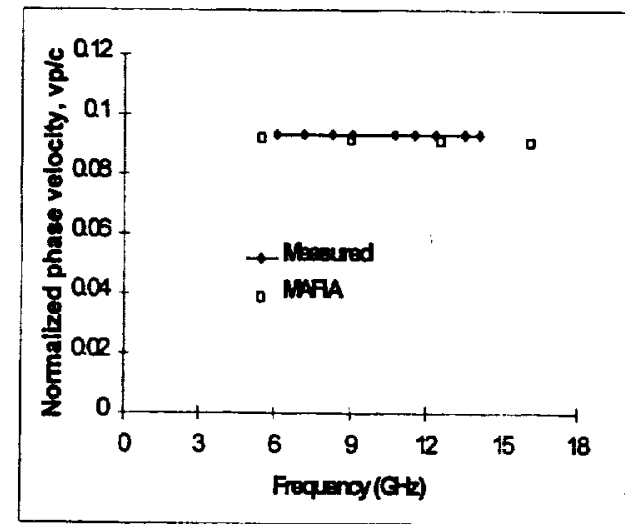

Figure 12 Measured and simulated dispersion for Hughes MPM helical circuit

\section{CONCLUSIONS}

An accurate three-dimensional TWT helical slow-wave circuit model has been introduced and its accuracy validated by comparing measured cold-test results with those obtained using MAFIA with excellent agreement. First an in depth analysis was given describing how MAFIA 
can be used to obtain the cold-test characteristics including dispersion, on-axis interaction impedance and attenuation. Then, the simulated results for several typical TWT helical slowwave structures were presented. The cold-test data from this helical model can be used as input into large-signal helical TWT interaction codes such as [16] making it possible, for the first time, to design a complete TWT via computer simulation.

The Northrop Grumman C-Band helical circuit, representing one of the most basic helical slow-wave structure designs, was modeled first. The cold-test results using MAFIA showed excellent agreement when compared to measured values with absolute average differences across the bandwidth of $0.7 \%$, six Ohms and $.0005 \mathrm{~dB} /$ turn, for the dispersion, impedance and attenuation, respectively.

Next the cold-test characteristics for the Northrop Grumman broadband helical circuit were presented and compared to measured data with absolute average differences across the bandwidth of $1.5 \%$, ten $\mathrm{Ohms}$ and $.003 \mathrm{~dB} / \mathrm{turn}$, for the dispersion, impedance and attenuation, respectively.

Two additional helical slow-wave circuits were modeled using MAFIA, the Ka-Band circuit from the TWTA for the Cassini mission, and the Hughes MPM circuit embodying T-shaped support rods. When the results for the Cassini circuit using MAFIA were compared to measured data, an absolute average difference across the operating range of $0.6 \%$ was found for the dispersion. The conductivity value used in the MAFIA simulation for the helical tape was obtained by matching the measured attenuation with experimental data. The conductivity for the helical tape was found to be $2.25 \times 10^{7} \mathrm{~S} / \mathrm{m}$, very close to the theoretical value of copper, indicating a very smooth helical surface. An absolute average difference of $1.8 \%$ across the operating range was obtained for the dispersion for the Hughes MPM helical circuit. 
A discrepancy in the agreement between the on-axis interaction impedance supplied from industry and that calculated using MAFIA is expected. The rnethods used to calculate this coldtest parameter differ. To experimentally obtain on-axis in:eraction impedance, the circuit is perturbed with a small dielectric rod at the center of the helical axis, and the change in phase shift between the perturbed and unperturbed circuit at a constant driving frequency is measured. Slater's perturbation theory [17] is used, along with several approximations for the fields within the circuit, to derive the formula for on-axis interaction impredance. Future work will involve investigating experimental methods of obtaining impedance to determine whether the impedance results obtained using MAFIA are more accurate than those obtained by experimentally perturbing the circuit. 


\section{LIST OF FIGURES}

Figure 1 Phase velocity versus frequency for several circuit sections of varying number of turns for the Northrop C-band helical circuit. 4

Figure 2 Northrop C-Band helical TWT slow-wave circuit ........................................................

Figure 3 MAFIA three-dimensional plot of Northrop C-Band helical circuit.................................

Figure 4 Measured and simulated dispersion and attenuation for Northrop C-Band helical circuit8

Figure 5 Measured and simulated on-axis interaction impedance for Northrop C-Band helical

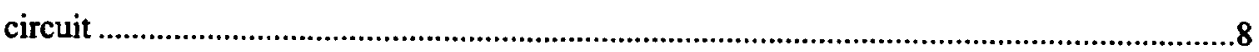

Figure 6 Northrop broadband helical TWT slow-wave circuit .................................................10

Figure 7 Northrop broadband helical slow-wave circuit model with graded wedge support rods 10

Figure 8 Measured and simulated dispersion and attenuation for Northrop broadband helical

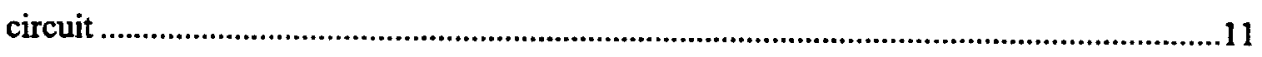

Figure 9 Measured and simulated on-axis interaction impedance for Northrop broadband helical circuit 11

Figure 10 Measured and simulated dispersion and attenuation for Cassini helical circuit............13

Figure 11 Hughes MPM helical slow-wave circuit ..................................................................14

Figure 12 Measured and simulated dispersion for Hughes MPM helical circuit..........................14 


\section{REFERENCES}

1. Kory, C. L., Three-Dimensional Simulation of Helix Traveling-Wave Tube Cold-Test Characteristics Using MAFIA, IEEE Trans. on Electron Devices, vol. 43, no. 8, pp. 1317-1319, August 1996.

2 K. D. Ward and J Wlodarczyk, Transmission line modelling of helix slow wave structures, 1993 IEDM Technical Digest, p. 157, IEEE 1993.

3. T. Weiland, On the Numerical Solution of Maxwell's Equations and Applications in the Field of Accelerator Physics, Part. Accel., vol 15, pp. 245-292, 1984.

4. T. Weiland, On the Unique Numerical Solution of Maxwellian Eigenvalue Problems in Three Dimensions, Part. Accel., vol. 17, pp. 227-242, 1985.

5 F. Kantrowitz and I. Tammaru, Three-Dimensional Simulations of Frequency-Phase Measurements of Arbitrary Coupled-Cavity RF Circuits, IEFE Trans. Electron Devices, vol. 35, no. 11, pp. 2018-2026, Nov. 1988.

6 E. J. Nalos, Measurement of Circuit Impedance of Periodically Loaded Structures by Frequency Perturbation, Proc. IRE, Vol. 42, pp. 1508-1511, Oct. 1954.

7 W. H. Watson and J. R. Whinnery, Study of a Plane Short On A Shielded Helix, IRE Trans. Electron Devices, vol. 2, Oct. 1955.

8. J. W. Gewartowski and H. A. Watson, Principles of Electron Tubes. D. Van Nostrand Company, Inc. p. 357, 1965.

9. O. P. Ghandi, Microwave Engineering and Applications. Pergamon Press, Elmsford, New York, 1981. 
10. Personal communication with Gary Groshart of Northrop Grumman Corp., Electronics Systems Division, Rolling Meadows, IL, USA.

11. A. S. Gilmour, Jr., M. R. Gillette and J-T. Chen: Theoretical and Experimental TWT Helix Loss Determination, IEEE Trans. on Electron Devices, vol. 26, no. 10, pp. 1581-1588, October 1979.

12. C. L. Kory, Validation Of An Accurate Three-Dimensional Helical Slow-Wave Circuit Model, NASA Contractor Report, CR-4766, March 1997.

13. A. N. Curren et al., A High Efficiency, Low Power Ka-Band TWTA for Cassini, 1994 IEDM Technical Digest, pp. 783-786, IEEE 1994.

14. S. Alterovitz et al., Characterization of Commercial APBN, NASA Technical Paper in preparation, 1997.

15. Personal communication with Ron LeBorgne of Hughes Aircraft Co., Electron Dynamics Division, Torrance, CA, USA.

16 H. K. Detweiler, Characteristics of magnetically focused large-signal traveling-wave amplifiers, Rome Air Development Center Tech. Rep. RADC-TR-68-433, Griffiss Air Force Base, NY, 1968.

17. J. C. Slater, Microwave Electronics. Van Nostrand, New York, 1950. 
